\title{
Avaliação da resistência de híbridos de milho ao ataque de Sitophilus zeamais motschulsky (Coleoptera: Curculionidae) no grão armazenado
}

\author{
Evaluation of resistance of corn hybrids against the attack of \\ Sitophilus zeamais motschulsky (Coleoptera: Curculionidae) \\ in stored kernels
}

\author{
Alberto Luiz Marsaro Júnior ${ }^{1 *}$; Sonia Maria Noemberg Lazzari²; Patrícia \\ Kadozawa $^{3}$; Elisa Yoko Hirooka ${ }^{4}$; Antônio Carlos Gerage ${ }^{5}$
}

\section{Resumo}

\begin{abstract}
A resistência de híbridos de milho, cultivados ou em fase de melhoramento genético no Paraná, foi avaliada com relação ao ataque de Sitophilus zeamais, principal praga de grãos armazenados de milho. Amostras de grãos de onze híbridos de milho, IPD T1/3AC, IPD 029, IPT 4/029, AG 5011, IPS G/58, IPT $2 /$ 15, IPT 4/15, IPT T/23, IPT 5/T02, IPT 2/92 e IPT 9/13, foram infestadas com adultos de $S$. zeamais e mantidas em BOD, a $27 \pm 1^{\circ} \mathrm{C}, 75 \pm 5 \%$ UR e 12 horas de fotofase. Após trinta dias da infestação foram avaliados os seguintes parâmetros de resistência: índice de suscetibilidade, perda de massa seca dos grãos, número de insetos emergidos, ciclo biológico e massa dos adultos. Os híbridos mais suscetíveis ao ataque de S. zeamais foram: IPD T1/3AC e IPD 029, enquanto que os mais resistentes foram: IPT 4/ 029, AG 5011, IPS G/58, IPT 2/15 e IPT 4/15.
\end{abstract}

Palavras-chave: Insecta, resistência de plantas, milho armazenado, gorgulho-do-milho

\begin{abstract}
Resistance of corn hybrids, cultivated or in phase of genetic improvement in Paraná, was evaluated against the attack of Sitophilus zeamais, main grain pest of corn storage. Samples of kernels of eleven hybrids of corn, IPD T1/3AC, IPD 029, IPT 4/029, AG 5011, IPS G/58, IPT 2/15, IPT 4/15, IPT T/23, IPT 5/ T02, IPT 2/92 and IPT 9/13, were infested with adults of S. zeamais and maintained in BOD, at $27 \pm 1^{\circ} \mathrm{C}$, $75 \pm 5 \% \mathrm{RH}$ and 12 hours of photophase. After 30 days of infestation the following parameters of resistance were evaluated: susceptibility index, reduce of dry mass of the grains, number of emerged insects, biological cycle and adult mass. The most susceptible hybrids to the attack of $S$. zeamais were: IPD T1/ 3 AC and IPD 029; while the most resistant were: IPT 4/029, AG 5011, IPS G/58, IPT 2/15 and IPT 4/15.
\end{abstract}

Key words: Insecta, plant resistance, stored corn, maize weevil

\footnotetext{
1 Entomologista Embrapa Roraima, Br 174, Km 8, Distrito Industrial, 69301-970, CP 133, Boa Vista/RR, alberto@cpafrr.embrapa.br.

2 Depto. de Zoologia, Universidade Federal do Paraná, Jardim das Américas, 81531-980, CP 19020, Curitiba/PR, lazzari@ufpr.br.

${ }^{3,4}$ Depto. Tecnologia Alimentos e Medicamentos, Universidade Estadual de Londrina, 86051-990, Londrina/PR, patriciakadozawa@hotmail.com,hirooka@uel.br.

5 Instituto Agronômico do Paraná, Londrina/PR, milhoger@pr.gov.br.

* Autor para correspondência.
} 


\section{Introdução}

O milho, Zea mays L. (Poaceae), é cultivado na maioria dos países, representando o segundo cereal mais importante em termos de produção mundial, suplantado apenas pelo trigo. Os Estados Unidos, a China e o Brasil figuram como os principais países produtores e, também, como os maiores consumidores. No Brasil, cerca de $45 \%$ da produção de milho destina-se para a indústria de ração de aves e suínos (GODOY, 1999).

Diante da necessidade de garantir o abastecimento de milho para sua utilização ao longo do ano, prevenir eventuais períodos de escassez, proporcionar maior estabilidade dos preços e preservar as qualidades físico-químicas e nutricionais dos grãos, faz-se necessário o armazenamento dos mesmos (FAO, 1985). Vale ressaltar que, mesmo sob boas condições de armazenamento, podem ocorrer perdas causadas por insetos, fungos, ácaros, roedores e pássaros (LAZZARI, 1997).

Dentre os insetos, o gorgulho-do-milho, Sitophilus zeamais Motschulsky (Coleoptera: Curculionidae), destaca-se como a principal praga do milho armazenado, devido ao seu elevado potencial biótico, capacidade de atacar grãos tanto no campo quanto em silos e de sobreviver a grandes profundidades na massa dos grãos (FARONI, 1992). Os principais prejuízos causados por $S$. zeamais são a perda de massa e do poder germinativo das sementes induzindo à desvalorização comercial dos grãos (LAZZARI, 1997). Além disso, favorece o desenvolvimento de fungos de armazenamento como o Aspergillus flavus e a conseqüente produção de aflatoxinas (BETI; PHILLIPS; SMALLEY, 1995).

Em decorrência dos prejuízos causados por $S$. zeamais e por outras pragas, faz-se necessária a aplicação contínua de medidas de controle. No Brasil utilizam-se, principalmente, os inseticidas protetores e fumigantes. Porém, devido ao seu uso indiscriminado, tem havido problemas com populações de insetos resistentes e com a contaminação de alimentos com resíduos de ingredientes ativos (LORINI, 2001).
A seleção de plantas com características de resistência a insetos apresenta-se como uma medida alternativa ao controle químico, inclusive para grãos armazenados. Alguns estudos têm demonstrado a existência de genótipos de milho resistentes a insetos durante o armazenamento (GÓMEZ; SANTOS; LIMA, 1994; BOIÇA JÚNIOR; LARA; GUIDI, 1997; TOSCANO et. al., 1999; CANEPPELE; CANEPPELE; LAZZARI, 2003).

A utilização de plantas resistentes a insetos apresenta uma série de vantagens com relação ao uso dos inseticidas químicos: não onera o custo de produção, não oferece riscos para a saúde humana e animal, reduz perdas quantitativas e qualitativas, não polui o meio ambiente e é compatível com outras estratégias de controle (LARA, 1991; MAZZONETO; BOIÇA JÚNIOR, 1999).

A resistência de grãos de milho ao ataque por $S$. zeamais tem sido relacionada com a presença de proteínas, lipídios, compostos fenólicos e inibidores de enzimas digestivas. Classen et al. (1990) verificaram que o teor de proteínas se correlacionava negativamente com o número de ovos nos grãos e com a progênie produzida. Arnason et al. (1993) constataram que o teor de lipídios se correlacionava negativamente com o índice de suscetibilidade e com o número de ovos nos grãos. Serratos et al. (1993) verificaram que o teor de compostos fenólicos se correlacionava negativamente com a progênie produzida e positivamente com o ciclo biológico.

Alguns estudos indicam que os inibidores de amilase de insetos têm um papel importante na resistência de híbridos de milho ao ataque de pragas de armazenamento. Marsaro Júnior (2004) verificou que inibidores de amilase de $S$. zeamais, presentes em grãos de híbridos de milho, entre eles o IPS G/58, correlacionaram-se negativamente com o índice de suscetibilidade e positivamente com o ciclo biológico. Figueira et al. (2003) e Kadozawa (2004), estudando o híbrido AG 5011, verificaram que inibidores de amilase, presentes nos grãos desse híbrido, inibiram as amilases de $S$. zeamais e Fusarium verticillioides. 
A consistência também é um dos fatores apontados como característica de resistência dos grãos contra o ataque de insetos. Porém, estudos que avaliaram a resistência de grãos duros e moles com relação ao ataque de pragas de armazenamento demonstraram que não houve diferença na performance dos insetos entre os dois grupos estudados (GÓMEZ; SANTOS; LIMA, 1994; SILVA et. al., 1998).

Esta pesquisa foi proposta com o objetivo de avaliar a resistência de híbridos de milho, cultivados ou em fase de melhoramento genético no Paraná, com relação ao ataque de $S$. zeamais durante o armazenamento do grão.

\section{Material e Métodos}

Inicialmente, plantou-se no campo experimental do IAPAR de Londrina/PR sementes dos híbridos IPD 0/29, IPS G/58, IPD T1/3AC, IPT T/23, IPT 5/ T02, IPT 2/92, IPT 9/13, IPT 4/15, IPT 2/15, IPT 4/ 029 e AG 5011, todos pertencentes ao grupo semiduro e classe amarelo excetuando-se o IPD 0/29 que é do grupo duro. Com exceção do híbrido AG 5011 que foi cedido pela AGROCERES, todos os demais são materiais do IAPAR. Todos os tratos culturais convencionais foram realizados durante todo o ciclo da cultura.

Após a colheita, amostras de aproximadamente $1 \mathrm{~kg}$ de grãos de cada híbrido, com umidade inicial em torno de $15 \%$, foram secas à sombra até atingirem $13,5 \%$ de umidade. Para a confirmação desta umidade três amostras de $5 \mathrm{~g}$ de grãos, de cada híbrido, foram colocadas em estufa a $105 \pm 3^{\circ} \mathrm{C}$, durante 24 horas (WAQUIM, 1992). Posteriormente, os grãos foram armazenados em sacos plásticos, a $20^{\circ} \mathrm{C}$, por 30 dias, a fim de eliminar os insetos provenientes do campo (FALEIRO et al., 1995).

Os indivíduos adultos de $S$. zeamais utilizados nos bioensaios foram obtidos na EMBRAPA Milho e Sorgo de Sete Lagoas, MG. Os insetos foram criados em potes de $2 \mathrm{~L}$ contendo grãos de milho limpo e seco (previamente congelados para eliminar infestações) e mantidos em temperatura ambiente no Laboratório de Entomologia da Universidade Federal do Paraná, Curitiba, PR.

A unidade experimental foi formada por uma amostra de $100 \mathrm{~g}$ de grãos de milho e por 15 fêmeas e 5 machos de $S$. zeamais, com idade entre 7 e 14 dias, por 10 dias, para a cópula e oviposição (DOBIE, 1977). Após esse período, os insetos adultos foram descartados. Cada amostra foi armazenada em recipiente plástico de $500 \mathrm{~mL}$ com tampa telada, em BOD, a $27 \pm 1{ }^{\circ} \mathrm{C}, 75 \pm 5 \%$ UR e 12 horas de fotofase (CANEPPELE; CANEPPELE; LAZZARI, 2003).

Decorridos trinta dias após a infestação avaliouse, a cada dois dias, o número de adultos emergidos, os quais eram descartados após a contagem. Essa avaliação foi realizada até o momento em que não mais se observou a emergência de gorgulhos adultos. Também foram avaliadas a massa dos gorgulhos adultos e a perda de massa seca dos grãos, provocada pelos indivíduos adultos e sua progênie. A pesagem dos adultos foi realizada após 24 horas de emergência (TOSCANO et. al., 1999). A perda de massa seca dos grãos foi determinada pela diferença entre a massa seca inicial e a massa seca final.

A resistência dos híbridos de milho foi avaliada pelo índice de suscetibilidade (IS) (DOBIE, 1977), que relaciona o número de gorgulhos emergidos e o tempo médio de desenvolvimento, após infestação artificial de grãos por $S$. zeamais. Esse índice foi determinado pelas seguintes equações: IS $=\left(\ln \left(\sum x\right) / T\right)$ x 100 (Equação 1); onde: IS = Índice de suscetibilidade; $\ln$ = logarítmo neperiano; $\sum x=$ somatório do número de gorgulhos emergidos em cada híbrido e; $\mathrm{T}=$ tempo médio gasto para os gorgulhos completarem o ciclo biológico. $\mathrm{T}=\sum(x y) / \sum x$ (Equação 2 ); onde: $x=$ número de gorgulhos emergidos diariamente e; $y=$ número de dias da infestação à emergência.

Os onze híbridos foram estudados em delineamento experimental de blocos casualizados, com três repetições. Os dados foram submetidos à 
análise de variância e, quando se detectaram diferenças significativas pelo teste $\mathrm{F}$, as médias foram comparadas pelo teste de Tukey no nível de 5\%.

\section{Resultados e Discussão}

Os grãos dos milhos híbridos IPD T1/3AC e IPD 029 , respectivamente, apresentaram os maiores índices de suscetibilidade $(13,57$ e 12,59) ao ataque por S. zeamais (Tabela 1); as maiores médias para a variável gorgulhos emergidos $(198,00$ e 165,33) (Tabela 1) e as maiores perdas de massa seca $(6,25$ g e 6,10 g) (Tabela 2). Também foi observado que o ciclo biológico de $S$. zeamais foi mais curto nesses híbridos (38,87 dias e 40,52 dias) (Tabela 1), mostrando que eles foram os mais adequados para a oviposição e/ou desenvolvimento das larvas. Por isso, esses híbridos quando infestados com o gorgulhodo-milho permitirão que exista número maior de gerações do inseto, quando comparados com os híbridos mais resistentes. Em conseqüência disso, apresentarão os maiores danos qualitativos e quantitativos.

Os resultados encontrados nesta pesquisa estão de acordo com os encontrados por Gómez, Santos e Lima (1994). Os autores verificaram que os híbridos de milho mais suscetíveis ao ataque de $S$. zeamais apresentaram valores para os índices de suscetibilidade acima de doze e ciclo biológico abaixo de quarenta e dois dias.
Os resultados encontrados para o número gorgulhos emergidos nos híbridos mais suscetíveis, IPD T1/3AC e IPD 029, 198,00 e 165,33, respectivamente, também estão de acordo com os valores encontrados por Serratos et al. (1993). Os autores verificaram que no híbrido mais suscetível ao ataque do gorgulho o número de adultos emergidos foi superior a cento e quarenta.

Os híbridos IPT 4/029 (IS = 9,44), AG 5011 (IS = 9,49), IPS G/58 (IS = 9,52), IPT 2/15 (IS = 9,95) e IPT 4/15 (IS = 10,12) apresentaram maior resistência ao ataque de $S$. zeamais (Tabela 1) e estão presentes no grupo dos híbridos que apresentaram as menores médias para as variáveis gorgulhos emergidos (Tabela 1) e para perda de massa seca dos grãos (Tabela 2). Os insetos desenvolvidos nos grãos desses híbridos também apresentaram as maiores médias para ciclo biológico (Tabela 1). Os resultados encontrados nesta pesquisa estão de acordo com os encontrados por Gómez, Santos e Lima (1994) e Serratos et al. (1993). Esses autores verificaram que os híbridos mais resistentes ao ataque de $S$. zeamais apresentaram valores para o índice de suscetibilidade abaixo de onze.

O ciclo biológico dos híbridos mais resistentes ao ataque de $S$. zeamais encontrados nesta pesquisa sempre foi superior a quarenta e quatro dias, semelhantemente ao encontrado por Gómez; Santos e Lima (1994).

Tabela 1. Médias \pm erro padrão do índice de suscetibilidade, ciclo biológico (dias) e gorgulhos emergidos, obtidos em amostras de $100 \mathrm{~g}$ de grãos de 11 híbridos de milho $\left(27 \pm 1^{\circ} \mathrm{C}, 75 \pm 5 \%\right.$ UR e 12 horas de fotofase).

\begin{tabular}{cccc}
\hline Híbridos & Índice de suscetibilidade & Ciclo biológico & Gorgulhos emergidos \\
\hline IPD T1/3AC & $13,57 \pm 0,20 \mathrm{a}$ & $38,87 \pm 0,23 \mathrm{e}$ & $198,00 \pm 20,43 \mathrm{a}$ \\
IPD 029 & $12,59 \pm 0,17 \mathrm{ab}$ & $40,52 \pm 0,47 \mathrm{de}$ & $165,33 \pm 10,68 \mathrm{ab}$ \\
IPT T/23 & $11,48 \pm 0,20 \mathrm{bc}$ & $43,38 \pm 0,19 \mathrm{c}$ & $147,33 \pm 14,17 \mathrm{abc}$ \\
IPT 5/T02 & $11,27 \pm 0,48 \mathrm{~cd}$ & $42,75 \pm 0,81 \mathrm{~cd}$ & $124,66 \pm 14,25 \mathrm{bcd}$ \\
IPT 2/92 & $10,94 \pm 0,45 \mathrm{cde}$ & $42,81 \pm 0,66 \mathrm{c}$ & $110,66 \pm 16,76 \mathrm{bcd}$ \\
IPT 9/13 & $10,78 \pm 0,11 \mathrm{cde}$ & $44,06 \pm 0,42 \mathrm{bc}$ & $117,00 \pm 10,15 \mathrm{bcd}$ \\
IPT 4/15 & $10,12 \pm 0,10 \mathrm{def}$ & $45,71 \pm 0,71 \mathrm{ab}$ & $102,33 \pm 5,36 \mathrm{~cd}$ \\
IPT 2/15 & $9,95 \pm 0,08 \mathrm{ef}$ & $44,65 \pm 0,24 \mathrm{abc}$ & $85,66 \pm 4,98 \mathrm{~d}$ \\
IPS G/58 & $9,52 \pm 0,09 \mathrm{f}$ & $45,69 \pm 0,26 \mathrm{ab}$ & $78,00 \pm 4,93 \mathrm{~d}$ \\
AG 5011 & $9,49 \pm 0,11 \mathrm{f}$ & $45,98 \pm 0,19 \mathrm{ab}$ & $79,00 \pm 5,57 \mathrm{~d}$ \\
IPT 4/029 & $9,44 \pm 0,06 \mathrm{f}$ & $46,62 \pm 0,15 \mathrm{a}$ & $81,66 \pm 1,20 \mathrm{~d}$ \\
\hline CV\% & 3,70 & 1,79 & 16,84 \\
\hline
\end{tabular}

As médias de cada variável seguidas pela mesma letra, nas colunas, não diferem entre si, pelo teste de Tukey, no nível de 5\%. 
Figueira et al. (2003) e Marsaro Júnior (2004) verificaram, respectivamente, que os híbridos $A G$ 5011 e IPS G/58 apresentaram 93,10 e 19,26 unidades inibidoras de amilase de $S$. zeamais/g de milho. Esses resultados indicam que os inibidores de amilase constituem um fator importante para a resistência desses híbridos contra o ataque de $S$. zeamais.

Com relação à massa dos gorgulhos adultos, constatou-se que não houve diferença significativa para esta variável (Tabela 2). Marsaro Júnior (2004) também não constatou diferença de massa entre os adultos desenvolvidos em diferentes híbridos de milho. Arnason et al. (1993) mencionaram que os níveis de proteínas nos híbridos atuais já são altos e geralmente estão acima do ótimo para o desenvolvimento de $S$. zeamais. Baseado nisso, é provável que todos os híbridos utilizados nesta pesquisa apresentem os requerimentos nutricionais básicos para o desenvolvimento de S. zeamais, o que justificaria a igualdade de peso dos gorgulhos adultos nos diferentes híbridos.

Tabela 2. Médias \pm erro padrão da perda de massa seca dos grãos e massa dos gorgulhos adultos, obtidos em amostras de $100 \mathrm{~g}$ de grãos de 11 híbridos de milho $\left(27 \pm 1^{\circ} \mathrm{C}, 75 \pm 5 \%\right.$ UR e 12 horas de fotofase).

\begin{tabular}{ccc}
\hline Híbridos & Perda de massa seca dos grãos $(\mathrm{g})$ & Massa dos gorgulhos adultos (mg) \\
\hline IPD 029 & $6,25 \pm 0,67 \mathrm{a}$ & $3,10 \pm 0,02 \mathrm{a}$ \\
IPD T1/3AC & $6,10 \pm 0,84 \mathrm{ab}$ & $2,98 \pm 0,04 \mathrm{a}$ \\
IPT T/23 & $5,70 \pm 0,80 \mathrm{abc}$ & $3,04 \pm 0,01 \mathrm{a}$ \\
IPT 9/13 & $4,32 \pm 0,33 \mathrm{abc}$ & $3,00 \pm 0,01 \mathrm{a}$ \\
IPT 2/15 & $4,30 \pm 0,18 \mathrm{abc}$ & $3,04 \pm 0,05 \mathrm{a}$ \\
IPT 5/T02 & $4,21 \pm 0,39 \mathrm{abc}$ & $3,08 \pm 0,01 \mathrm{a}$ \\
IPT 4/15 & $3,95 \pm 0,24 \mathrm{abc}$ & $2,99 \pm 0,04 \mathrm{a}$ \\
IPS G/58 & $3,59 \pm 0,34 \mathrm{bc}$ & $3,06 \pm 0,02 \mathrm{a}$ \\
AG 5011 & $3,53 \pm 0,30 \mathrm{bc}$ & $2,96 \pm 0,02 \mathrm{a}$ \\
IPT 4/029 & $3,49 \pm 0,12 \mathrm{bc}$ & $2,98 \pm 0,03 \mathrm{a}$ \\
IPT 2/92 & $3,33 \pm 0,81 \mathrm{c}$ & $3,06 \pm 0,01 \mathrm{a}$ \\
\hline CV\%
\end{tabular}

As médias de cada variável seguidas pela mesma letra, nas colunas, não diferem entre si, pelo teste de Tukey, no nível de $5 \%$.

\section{Conclusões}

Todos os híbridos de milho avaliados nesta pesquisa apresentaram um certo grau de suscetibilidade ao ataque de Sitophilus zeamais, porém nos híbridos mais resistentes ocorreu menor emergência de adultos, menor perda de massa seca dos grãos e maior período para que o inseto complete o ciclo biológico.

\section{Agradecimentos}

Ao pesquisador do Centro Nacional de Pesquisa de Milho e Sorgo (CNPMS) da EMBRAPA, Sete Lagoas, MG, Dr. Jamilton P. dos Santos, pela doação dos insetos e sugestões para a realização dos testes de resistência.

À Coordenação de Aperfeiçoamento de Pessoal de Nível Superior (CAPES) pela concessão da bolsa de estudo para o primeiro autor.

Este estudo é a contribuição número 1516 do Departamento de Zoologia da Universidade Federal do Paraná. 


\section{Referências}

ARNASON, J. T.; LAMBERT, J. D. H.; GALE, J.; MIHM, J.; BJARNASON, M.; JEWELL, D.; SERRATOS, J. A.; FREGEAU-REID, J.; PIETRZAK, L. Is "quality protein” maize more susceptible than normal cultivars to attack by the maize weevil Sitophilus zeamais? Postharvest Biology and Technology, Amsterdam, v. 2, p. 349-358, 1993.

BETI, J. A.; PHILLIPS, T. W.; SMALLEY, E. B. Effects of maize weevils (Coleoptera: Curculionidae) on production of aflatoxin B sub (1) by Aspergillus flavus in stored corn. Journal of Economic Entomology, Lanham, v. 88, n. 6, p. 1776-1782, 1995.

BOIÇA JÚNIOR, A. L.; LARA, F. M.; GUIDI, F. P. Resistência de genótipos de milho ao ataque de Sitophilus zeamais (Mots.) (Coleoptera: Curculionidae). Anais da Sociedade Entomológica do Brasil, Jaboticabal, v. 26, n. 3, p. 481-485, 1997.

CANEPPELE, C.; CANEPPELE, M. A. B.; LAZZARI, S. M. N. Resistência de híbridos de milho, Zea mays (L.) ao ataque de Sitophilus zeamais (Mots.). Revista Brasileira de Armazenamento, Viçosa, v. 28, p. 51-58, 2003.

CLASSEN, D.; ARNASON, J. T.; SERRATOS, J. A.; LAMBERT, J. D. H.; NOZZOLILLO, C.; PHILOGÈNE, B. J. $\mathrm{R}$. Correlation of phenolic acid content of maize to resistance to Sitophilus zeamais, the maize weevil, in CIMMYT'S collections. Journal of Chemical Ecology, New York, v. 16, n. 2, p. 301-315, 1990.

DOBIE, P. The contribution of the tropical stored products centre to the study of insect resistance in stored maize. Tropical Stored Products Information, Berkshire, v. 34, p. 7-22, 1977.

FALEIRO, F. G.; PICANÇO, M.; MIRANDA, M. M. M.; ARAÚJO, J. M.; SARAIVA, L. S. Resistência de 49 populações de milho a Sitophilus zeamais Motsch. (Coleoptera: Curculionidae). Revista Brasileira de Armazenamento, Viçosa, v. 20, p. 17-21, 1995.

FAO. Prevención de pérdidas de alimentos pos-cosecha: manual de capacitación. Roma: ONU/FAO, 1985. 130 p.

FARONI, L. R. A. Manejo das pragas de grãos armazenados e sua influência na qualidade do produto final. Revista Brasileira de Armazenamento, Viçosa, v. 76, p. 36-43, 1992.

FIGUEIRA, E. L.Z.; HIROOKA, E. Y;; MENDIOLA-OLAYA, E.; BLANCO-LABRA, A. Characterization of an hydrophobic amylase inhibitor from corn (Zea mays) seeds with activity against amylase from Fusarium verticillioides. Phytopathology, St. Paul, v. 93, n. 8, p. 917-922, 2003.

GODOY, R. C. B. Acompanhamento da situação agropecuária do Paraná: prognóstico da cultura do milho. Curitiba: Secretaria de Estado da Agricultura, 1999. $42 \mathrm{p}$.
GÓMEZ, H. S.; SANTOS, J. P.; LIMA, J. O. G. Resistencia de genotipos de maíz con diferentes características físicas y químicas al ataque de Sitophilus zeamais Motschulsky (Coleoptera: Curculionidae). Revista Colombiana de Entomologia, Bogota, v. 20, n. 1, p. 37-42, 1994.

KADOZAWA, P. Inibidores de amilase e protease contra Fusarium verticillioides durante a germinação do milho. Londrina, 2004. 73 p. Dissertação (Mestrado em Ciência de Alimentos) - Universidade Estadual de Londrina, Londrina, 2004.

LARA, F. M. Princípios de resistência de plantas a insetos. São Paulo: Ícone, 1991. 335 p.

LAZZARI, F. A. Umidade, fungos e micotoxinas na qualidade de sementes, grãos e rações. Curitiba: Paranaset, 1997. $148 \mathrm{p}$.

LORINI, I. Manual técnico para o manejo integrado de pragas de grãos de cereais armazenados. Passo Fundo: Embrapa Trigo, 2001.80 p.

MARSARO JÚNIOR, A. L. Resistência de genótipos de milho, Zea mays L. (Poaceae), ao ataque de Sitophilus zeamais Motschulsky (Coleoptera: Curculionidae). 2004. 84 p. Tese (Doutorado em Entomologia) - Universidade Federal do Paraná, Curitiba, 2004.

MAZZONETO, F.; BOIÇA JÚNIOR, A. L. Determinação dos tipos de resistência de genótipos de feijoeiro ao ataque de Zabrotes subfasciatus (Boh.) (Coleoptera: Bruchidae). Anais da Sociedade Entomológica do Brasil, Jaboticabal, v. 28, n. 2, p. 307-311, 1999.

SERRATOS, J. A.; BLANCO-LABRA, A.; MIHM, J. A.; PIETRZAK, L.; ARNASON, J. T. Generation means analysis of phenolic compounds in maize grain and susceptibility to maize weevil Sitophilus zeamais infestation. Canadian Journal of Botany, Ottawa, v. 71, p. 1176-1181, 1993.

SILVA, A. A. L.; FARONI, L. R. D’ANTONINO; MARTINS, J. H.; CECON, P. R. Influência do processo de colheita na infestação do milho (Zea mays L.) pelo besouro da farinha (Tribolium castaneum Herbest) durante o armazenamento. Revista Brasileira de Engenharia Agrícola e Ambiental, Campina Grande, v. 2, n. 3, p. 247-352, 1998.

TOSCANO, L. C.; BOIÇA JÚNIOR, A. L.; LARA, F. M.; WAQUIL, J. M. Resistência e mecanismos envolvidos em genótipos de milho em relação ao ataque do gorgulho, Sitophilus zeamais Mots. (Coleoptera: Curculionidae). Anais da Sociedade Entomológica do Brasil, Jaboticabal, v. 28, p. 141-146, 1999.

WAQUIM, J. S. Regras para análise de sementes. Brasília: Ministério da Agricultura e Reforma Agrária, 1992. 365 p. 